\title{
Effect of different extraction solvents on the phenolic content and antioxidant activity of turmeric (Curcuma longa) from South-West Region, Cameroon
}

\author{
${ }^{2}$ Array, E.J., ${ }^{1 *}$ Tonfack Djikeng, F., ${ }^{2}$ Kingne Kingne, F., ${ }^{1}$ Kinge, E.E. and ${ }^{3}$ Womeni, H.M. \\ ${ }^{1}$ School of Agriculture and Natural Resources, Catholic University Institute of Buea, P.O BOX 563, Buea, \\ Cameroon \\ ${ }^{2}$ School of Engineering, Catholic University Institute of Buea, P.O BOX 563, Buea, Cameroon \\ ${ }^{3}$ Department of Biochemistry, Faculty of Science, University of Dschang, P.O BOX 67, Dschang, Cameroon
}

\author{
Article history: \\ Received: 13 September 2018 \\ Received in revised form: 29 \\ October 2018 \\ Accepted: 30 October 2018 \\ Available Online: 6 \\ November 2018
}

\section{Keywords:}

Phenolic content,

Antioxidant activity,

Solvents,

Curcuma longa

\section{DOI:}

https://doi.org/10.26656/fr.2017.3(1).227

\begin{abstract}
The objective of this study was to evaluate the effect of different solvent extraction on the phenolic content and antioxidant activity of turmeric (Curcuma longa). Fresh turmeric roots were dried, grounded and antioxidants extracted by maceration in methanol, ethanol, cold and warm water. The phenolic content of the extracts was determined using the FolinCiocalteu Method. A total of three antioxidant tests were done on the extracts: the 2, 2diphenyl-1-picrylhydrazyl test (DPPH test), the Ferric reducing antioxidant power (FRAP) and the Metal chelation ability (MCA). Results showed that the alcoholic extracts exhibited the highest phenolic content (TPC) (15.71-40.81mg GAE/g) and antioxidant activities (AA). However, the highest TPC (40.81mg GAE/g) and AA were recorded with the ethanolic extract ( 81.96 to $92.56 \%$ for the DPPH test; 0.88 to 2.12 for the FRAP; and 64.87 to $98.12 \%$ for the MCA each at concentrations 25 to $200 \mu \mathrm{g} / \mathrm{ml}$ ). Among the aqueous extracts, the phenolic content and antioxidant activity of the warm aqueous extract was significantly higher $(\mathrm{p}<0.05)$ than that of the cold aqueous extract. Ethanol is best for the extraction of phenolic antioxidant from turmeric compared to methanol and water. For local use, warm water can be recommended, as it extracts more antioxidants than cold water.
\end{abstract}

\section{Introduction}

In the recent years, the active principles provided by the secondary metabolism of plants such as herbs, spices, vegetables, fruits have been widely used to cure, inhibit or reduce the risk of human diseases. These secondary metabolites have several health benefits due to their antioxidant activity and their capacity to delay oxidative damages that have been proven to be implicated in several diseases such as cancer and cardiovascular disorder (Shahidi and Ambigaipalan, 2015). Among these active molecules, phenolic acids and flavonoids are the most represented. These bioactive compounds vary in type, number and position of functional groups, resulting in a variation of their activity. Plants that are generally rich in these compounds have very good antioxidant activity as they have the ability to stop or protect the biological system against the potentially harmful effects of free radicals and reactive oxygen species when there is imbalance between these reactive oxygen species and the natural antioxidant present in the body (Jakopic et al., 2009). Herbs and spices are generally used in the preparation of food mostly to improve in their color and flavour. They are also used as preservative agents to extend the shelf life of foods (Kivilompolo et al., 2007) or as an ingredient in the preparation of food and as traditional medicines. These plants have been reported to be rich in antioxidant components, mostly polyphenol which is responsible for many of their biological activities (Figueirinha et al., 2008). Because of the variability of the active constituents' present, the selection of the appropriate solvent for the maximal extraction of polyphenols is recommended, since these molecules are extracted at different levels by each solvent. It is important to know the best solvent for a good extraction of those active principles in such a way that it can be recommended for high or low scale industrial use. In previous studies, researchers have evaluated the effect of extraction methods using different solvents on the phenolic content and antioxidant activity of plant materials. In one study, Sepahpour et al. (2018) have demonstrated that different solvent extraction of turmeric was lead to different phenolic content and antioxidant activity. However, these authors limited their 
study on a specific concentration of organic solvents $(80 \%)$ and cold water. They did not evaluate the effect of extraction with warm water, $95 \%$ ethanol and $99 \%$ methanol on the phenolic content and antioxidant activity of Curcuma longa. Additionally, very few similar reports are available on Curcuma longa produced and commercialized in Cameroon. The use of different solvents for the extraction of active principles from Curcuma longa cultivated in Cameroon might have an impact on the bioactive molecule available and its antioxidant activity. The objective of this study was to evaluate the effect of different extraction methods on the phenolic content and antioxidant activity of turmeric rhizome.

\section{Materials and methods}

\subsection{Materials}

Dried turmeric (Curcuma longa) was purchased from Muea local market, Buea, South-West Region, Cameroon in May 2018. All chemicals and reagents used were of analytical reagent grade.

\subsection{Methods}

\subsubsection{Sample preparation and processing}

Fresh turmeric was sliced into smaller pieces and dried in an oven for $24 \mathrm{hrs}$ at $48^{\circ} \mathrm{C}$. The dried turmeric was then divided into four groups and allocated the following codes; T1, T2, T3 and T4. The turmeric of each group was then ground using a grinding machine (brand: Aifa, China).

\subsubsection{Extraction of turmeric phenols.}

The dried turmeric (T1, T2, T3, T4) each weighing $34.5 \mathrm{~g}$ was into four separate containers, and then extracted using the following; T1 cold water, T2 hot water, T3 ethanol and T4 methanol each at volume of $350 \mathrm{~mL}$, for $24 \mathrm{hrs}$ at room temperature, the mixtures were regularly subjected to shaking during the extraction. The extracts were then filtered with a Whatman No. 1 filter paper. The combined filtrates were subjected to rotatory evaporation at $40^{\circ} \mathrm{C}$ under reduced pressure to the removal of the solvent. The dried extract was used for the determination of total phenolic content and antioxidant activities.

\subsubsection{Effect of processing on the total polyphenol of turmeric.}

The effect of processing on the total phenolic content of turmeric was determined using the Folin-Ciocalteu colorimetric method, as described by Gao et al. (2000). In a test tube of $5 \mathrm{ml}$ volume, $20 \mu \mathrm{L}$ of a $2 \mathrm{mg} / \mathrm{mL}$ extract solution was added, followed by the FolinCiocalteu reagent $(0.2 \mathrm{~mL})$ and distilled water $(2 \mathrm{~mL})$.
After 3 mins incubation of the solution mixture at room temperature, $1 \mathrm{~mL}$ of $20 \%$ sodium carbonate solution was added and the mixture was re-incubated for 20 mins under the same conditions. The absorbance of the resulting blue coloured solution was measured at $765 \mathrm{~nm}$ using a spectrophotometer. The total phenolic content of the extract was calculated from the gallic acid standard curve and expressed as milligrams equivalents gallic acid per gram of extract.

\subsubsection{Effect of processing on the antioxidant activity of turmeric}

\subsubsection{DPPH radical scavenging assay}

The radical scavenging ability of the extracts was determined according to the method of Braca et al. (2002). Briefly, $4.5 \mathrm{~mL}$ of $0.002 \%$ alcoholic solution of DPPH was added to $0.5 \mathrm{~mL}$ of different concentrations $(125,250,500,1000$ and $2000 \mu \mathrm{g} / \mathrm{mL})$ of samples and standard solutions separately, in order to have final concentrations of products of $25-200 \mu \mathrm{g} / \mathrm{mL}$. The samples were kept at room temperature in the dark and after 30 mins, the absorbance of the resulting solution was measured at $517 \mathrm{~nm}$. The absorbance of the samples, control and blank was measured in comparison with methanol. Synthetic antioxidant, butylated hydroxytoluene (BHT), which is a recognized powerful hydrogen donor, was used as positive control. The antiradical activity (AA) was determined using the following formula:

$$
\mathrm{AA} \%=\left[\left(\mathrm{Abs}_{\text {control }}-\mathrm{Abs}_{\text {sample })} \times 100 / \mathrm{Abs}_{\text {control }}\right]\right.
$$

Where $A_{b s \text { control was the absorbance of control and } A_{b s}}$ sample the absorbance of the sample or standard.

\subsubsection{Ferric reducing antioxidant power}

The antioxidant potential of Curcuma longa extracts was also evaluated by their ability to reduce iron (III) to iron (II) following the method of Oyaizu (1986). An aliquot of $0.5 \mathrm{~mL}$ plant extract $(125,250,500,1000$ and $2000 \mu \mathrm{g} / \mathrm{mL}$ ) was mixed with $1 \mathrm{~mL}$ phosphate buffer (0.2 M, pH 6.6) and $1 \mathrm{~mL}$ of $1 \%$ aqueous $\mathrm{K}_{3} \mathrm{Fe}(\mathrm{CN})_{6}$ solution, well shaken and incubated at $50^{\circ} \mathrm{C}$ for 30 mins. After incubation, $1 \mathrm{~mL}$ of $10 \%$ TCA solution was added to stop the reaction and the mixture was centrifuged at $3000 \mathrm{rpm}$ for 10 mins. Briefly, $1.5 \mathrm{~mL}$ of supernatant, $1.5 \mathrm{~mL}$ of distilled water and $0.1 \mathrm{~mL}$ of $0.1 \% \mathrm{FeCl}_{3}$ solution were mixed and incubated for $10 \mathrm{mins}$ and absorbance read at $700 \mathrm{~nm}$ using a spectrophotometer. A sample blank, containing all the reagents but no extract was prepared in the same conditions. Catechin, a recognized powerful ferric reducer, was used as positive control to compare the reducing power of the extracts. A higher absorbance indicates a higher reducing power. 


\subsubsection{Metal chelation ability}

The antioxidant potential of Curcuma longa extracts was also evaluated by its ferrous ion chelating activity (Benzie and Szeto, 1999). In test tubes containing 160 $\mu \mathrm{L}$ of sample solution $(1000 \mu \mathrm{g} / \mathrm{mL}), 160 \mu \mathrm{L}$ of aqueous solution of 1,10 -phenanthroline $(0.25 \%)$ and $400 \mu \mathrm{L}$ of methanolic $\mathrm{FeCl}_{2}(0.1 \%)$ were added. After 10 mins incubation at room temperature, $880 \mu \mathrm{L}$ of distilled water was added and the absorbance was measured at $510 \mathrm{~nm}$. The metal chelating efficiency of the Curcuma longa extracts was compared to that of catechin (positive control). The inhibition percentage (IP) of the formation of the complex $\mathrm{Fe}^{2+}$-phenanthroline was calculated using the following formula:

$$
\mathrm{IP} \%=\text { Abs sample } \times 100 / \text { Abs control }
$$

\subsection{Statistical analysis}

Results (Mean \pm Standard deviation) obtained in the present study were subjected to one-way analysis of variance (ANOVA) with Student-Newman- Keuls test using Graphpad-InStat version 3.05, to evaluate the statistical significance of the data. A probability value at $\mathrm{p}<0.05$ was considered statistically significant.

\section{Results and discussion}

\subsection{Results}

\subsubsection{Total phenolic content}

The total phenolic contents of Curcuma longa rhizomes extracted using different solvents and conditions are presented in Figure 1. The ethanolic extract has exhibited significantly higher $(\mathrm{p}<0.001)$ phenolic content (40.80 mg GAE/g) compared to the other samples. It was followed by the methanolic extract (15.71 mg GAE/g) and the warm aqueous extract (7.54 $\mathrm{mg}$ GAE/g). The lowest phenolic content was registered with the cold aqueous extract (4.79 $\mathrm{mg} \mathrm{GAE} / \mathrm{g}$ ).

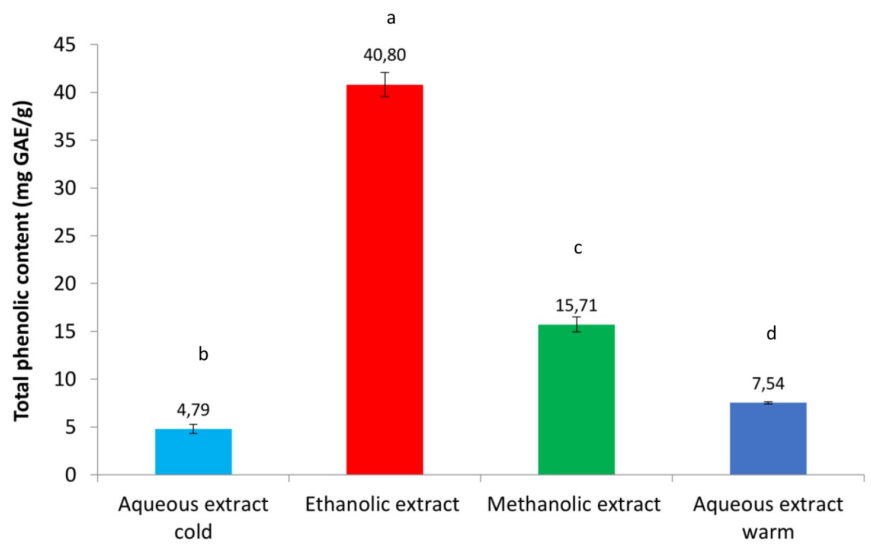

Figure 1. Total phenolic content of different extracts of turmeric. Values are presented as mean and standard deviation $(n=3)$. Means with different superscripts alphabets are significantly different $(\mathrm{p}<0.05)$.

\subsubsection{Antioxidant activity}

\subsubsection{Radical scavenging activity}

The radical scavenging activity of different turmeric extracts is illustrated in Figure 2. It is clearly observed that the ethanolic extract of this plant has exhibited significantly higher $(\mathrm{p}<0.05)$ activity $(80-90 \%)$ compared to the other extracts and this at all concentrations. It was followed by the methanolic extract (53-85\%), the warm aqueous extract (15-60\%) and the cold aqueous extract (15-35\%). Generally, apart from the ethanolic extract which exhibited similar activity $(p>0.05)$ at all concentrations, the activity of the other extracts was significantly different $(p<0.05)$ at the concentration.

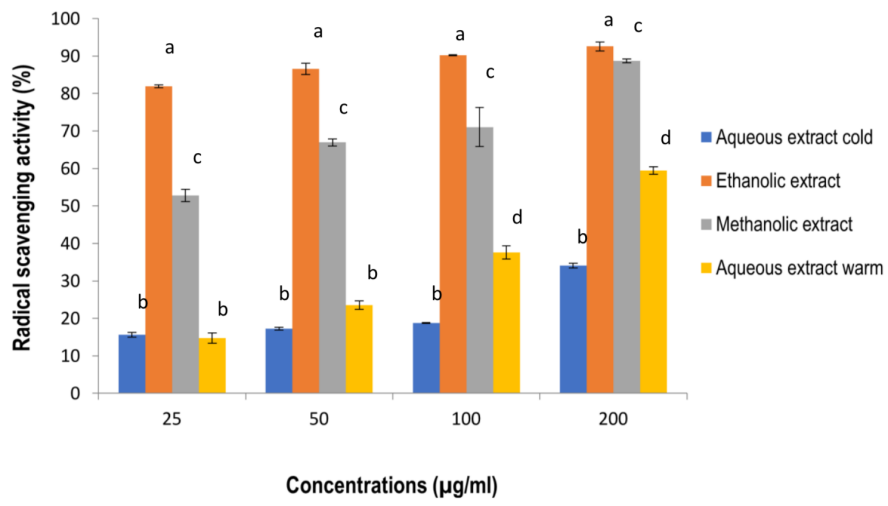

Figure 2. Radical scavenging activity of different extracts of turmeric. Means with different superscripts alphabets are significantly different $(\mathrm{p}<0.05)$.

\subsubsection{Ferric reducing antioxidant power}

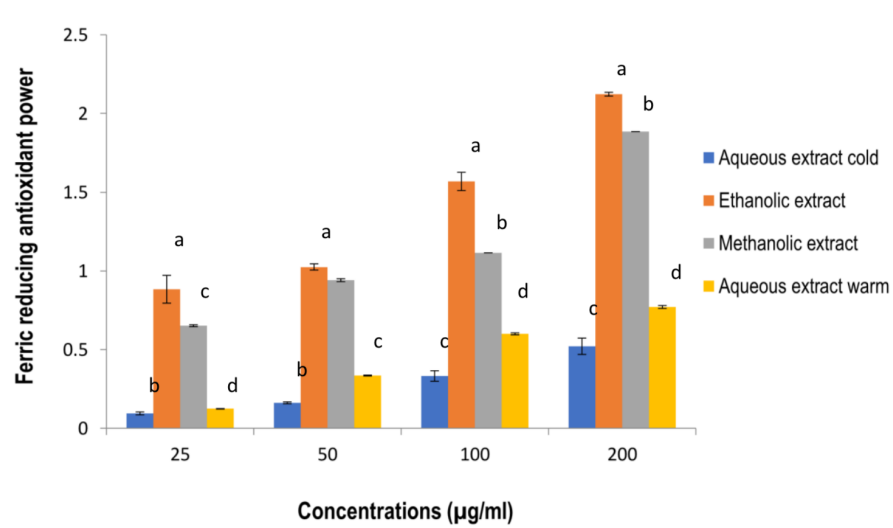

Figure 3. Ferric reducing antioxidant power of different extracts of turmeric. Values are presented as mean and standard deviation $(n=3)$. Means with different superscripts alphabets are significantly different $(\mathrm{p}<0.05)$.

The ferric reducing antioxidant power of Curcuma longa extracts is presented in Figure 3. As previously observed with the radical scavenging activity, the highest ferric reducing antioxidant power was registered with the ethanolic extract, followed by the methanolic extract. The activity of the cold and warm aqueous extract was significantly lower $(\mathrm{p}<0.05)$ compared to that of those of the extracts obtained using organic solvents. However, 
the ferric reducing power of the warm aqueous extract was significantly higher $(p<0.05)$ than that of the cold aqueous extract. Generally, the activity of all these extracts was increasing with their concentration.

\subsubsection{Metal chelation ability}

The ability of different extracts of Curcuma longa rhizome to chelate transition metals is presented in Figure 4. The ethanolic extract exhibited the highest $(p<0.001)$ metal chelation ability compared to the other extracts. Its activity was the best at all concentrations. This extract was followed by the methanolic extracts and warm aqueous extract. The lowest activity $(p<0.05)$ was registered with the cold aqueous extract. Generally, the activity of all these extracts was proportional to their concentrations.

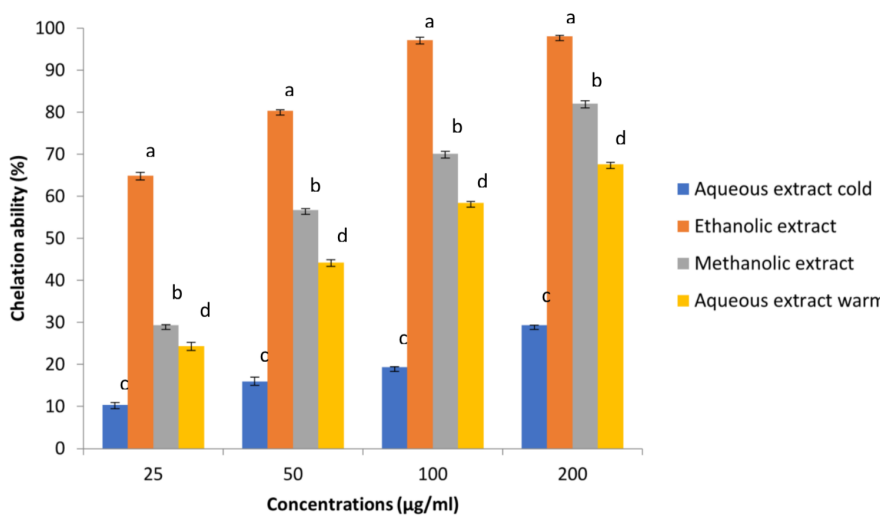

Figure 4. Metal chelation ability of different extracts of turmeric. Values are presented as mean and standard deviation $(n=3)$. Means with different superscripts alphabets are significantly different $(\mathrm{p}<0.05)$.

\section{Discussion}

The phenolic antioxidant extracted from plants have been intensively investigated for their biological activities among which their antioxidant activity. Many scientific reports have demonstrated that plant extracts contained a high amount of phenolic compound which possess good antioxidant activity (Abdou Bouba et al., 2010; Womeni et al., 2013). The total phenolic content of turmeric extracts analyzed using the Folin- Ciocalteu method showed that organic solvents (Ethanol and methanol) are suitable for the maximal extraction of the phenolic compound from turmeric rhizomes. The Total phenolic content of the warm aqueous extract was significantly higher than that of the cold aqueous extract. The differences observed in the phenolic content of these extracts can be attributed to the nature of the extracted solvent and the chemical properties of the phenolic antioxidants available. The fact that organic solvents generally exhibit good antioxidant activity than aqueous solvent has already been reported by Iqbal and Bhanger (2007) who demonstrated that the phenolic content of ethanolic and methanolic extracts of garlic was significantly higher than that of the aqueous and acetone extracts. The total phenolic content obtained in this study with the methanolic extract was $15.71 \mathrm{mg} \mathrm{GAE} / \mathrm{g}$. This value was significantly lower than that reported by Alafiatayo Akinola et al. (2014) who obtained a value of $43 \mathrm{mg} \mathrm{GAE} / \mathrm{g}$. However, the total phenolic content of Curcuma longa extracts obtained in this study was significantly higher than that reported by VallverduQueralt et al. (2015) who obtained a value of $9 \mu \mathrm{g} / \mathrm{g}$ with the hydroalcoholic extract of the same plant. The variations observed can be attributed to environmental differences, the harvesting period and the extraction and characterization methods (Shan et al., 2005). The high total phenolic content of the warm aqueous extract compared to the cold one can be explained by the fact that heat has facilitated the rupture and release of these active principles from plant cells. The results obtained in this study showed that the total phenolic content of the ethanolic and methanolic extracts was higher than those of the aqueous extracts. This result agrees with the findings of Sepahpour et al. (2018) who demonstrated that the total phenolic content of turmeric extracted with $80 \%$ acetone, $80 \%$ ethanol, $80 \%$ methanol and cold water were respectively $221.7,172.1,90.1$ and $3.8 \mathrm{mg}$ $\mathrm{GAE} / \mathrm{g}$ of freeze-dried crude extract.

The antioxidant activity of different extracts of Curcuma longa was also evaluated using different mechanisms of action. Results showed that the ethanolic and methanolic extracts exhibited the best antioxidant activity compared to aqueous extracts. This can be attributed to the high concentration in phenolic antioxidants. It has been demonstrated that phenolic antioxidants have the ability to donate their atoms for the stabilization of free radicals generated by the oxidative stress or to reduce and chelate transition metals that facilitate the generation of these radicals. This significantly depends on the structure of the antioxidant present. Ethanol and methanol might be having the power to extract more phenolic antioxidant with different mechanisms of action than warm and cold water. The result obtained in this study showed that organic solvents of turmeric have good antioxidant activity than aqueous solvent. This observation is in line with those reported by Sepahpour et al. (2018) who demonstrated that the radical scavenging activity of $80 \%$ acetone, $80 \%$ ethanol, $80 \%$ methanol and aqueous extract were respectively $67.8,47.4,27.8$ and $13.8 \%$. In a similar way, it was demonstrated that the ferric reducing antioxidant power of these same extracts were respectively $85.0,55.8,25.4$ and 2.3. Ethanol might be the best solvent to be used for a maximal extraction of molecules with good antioxidant activity from Curcuma longa rhizome. 


\section{Conclusion}

Results showed that the alcoholic extract has the highest phenolic content in antioxidant activity. However, the ethanolic extract was the best. Among the aqueous extracts, the phenolic content and antioxidant activity of the warm aqueous extract was significantly higher than that of the cold aqueous extract. Ethanol is the best solvent for the extraction of phenolic antioxidant compared to methanol and water. The ethanolic extract can be used for the extraction of active principles from Curcuma longa roots for medicinal purpose. In addition, at the local level, warm water can be recommended.

\section{References}

Alafiatayo Akinola A., Ahmad S. and Maziah M. (2014). Total Antioxidant Capacity, Total Phenolic Compounds and the Effects of Solvent Concentration on Flavonoid Content in Curcuma longa and Curcuma xanthorrhiza Rhizomes. Medicinal and Aromatic Plants, 3(2), 1-4. https:// doi.org/10.4172/2167-0412.1000156

Jakopic, J., Stampar, F. and Veberic, R. (2009). The influence of exposure to light on the phenolic content of 'Fuji' apple. Scientia Horticulturae, 123 (2), 234-239. https://doi.org/10.1016/ j.scienta.2009.09.004

Benzie, I.F.F and Szeto, Y.T. (1999). Total antioxidant capacity of teas by the ferric reducing/antioxidant power (FRAP) assay. Journal of Agricultural and Food Chemistry, 47(2), 633-636. https:// doi.org/10.1021/jf9807768

Abdou Bouba, A., Njintang, Y.N., Scher, J. and Mbofung, C.M.F. (2010) Phenolic compounds and radical scavenging potential of twenty Cameroonian spices. Agriculture and Biology Journal of North America, 1(3), 213-224. https://doi.org/10.5251/ abjna.2010.1.3.213.224

Braca, C., Sortino, M., Politi, I. and Morelli, J.M. (2002). Antioxidant activity of flavonoids from Licanialicaniaeflora. Journal of Ethnopharmacology, 79, 379-381. https:// doi.org/10.1016/S0378-8741(01)00413-5

Figueirinha, A., Paranhos, A., Perez-Alonso, J.J., SantosBuelga, C. and Batista, M.T. (2008). Cymbopogon citrates leaves: Characterisation of flavonoids by HPLC-PDA-ESI/MS/MS and an approach to their potential as a source of bioactive polyphenols. Food Chemistry, 110(3), 718-728. https://doi.org/10.1016/ j.foodchem.2008.02.045

Gao, X., Ohlander, M., Jeppsson, N., Björk, L. and Trajkovski, V. (2000). Changes in antioxidant effects and their relationship to phytonutrients in fruits of sea buckthorn (Hippophae rhamnoides L) during maturation. Journal of Agricultural and Food Chemistry, 48(5), 1485-1490.

Iqbal, S. and Bhanger, M.I. (2007). Stabilization of sunflower oil by garlic extract during accelerated storage. Food Chemistry, 100(1), 246-254. https:// doi.org/10.1016/j.foodchem.2005.09.049

Kivilompolo, M. and Hyotylainen, T. (2007). Comprehensive two-dimensional liquid chromatography in analysis of Lamiaceae herbs: characterisation and quantification of antioxidant phenolic acids. Journal of Chromatography A, 1145 (1-2), 155-164. https://doi.org/10.1016/ j.chroma.2007.01.090

Oyaizu, M. (1986). Studies on products of browning reaction: antioxidative activity of products of browning reaction. Japanese Journal of Nutrition, 44(6), 307-315. https://doi.org/10.5264/ eiyogakuzashi.44.307

Sepahpour, S., Selamat, J., Manap, M.Y.A., Khatib, A. and Razis, A.F.A (2018). Comparative Analysis of Chemical Composition, Antioxidant Activity and Quantitative Characterization of Some Phenolic Compounds in Selected Herbs and Spices in Different Solvent Extraction Systems. Molecules, 23 (2), 402. https://doi.org/10.3390/molecules 23020402

Shahidi, F. and Ambigaipalan, P. (2015). "Phenolics and polyphenolics in foods, beverages and spices: Antioxidant activity and health effects - A review." Journal of Functional Foods, 18(Part B), 820-897. https://doi.org/10.1016/j.jff.2015.06.018

Shan, B., Cai, Y.Z., Sun, M. and Corke, H. (2005). Antioxidant capacity of 26 spice extracts and characterization of their phenolic constituents. Journal of Agricultural and Food Chemistry, 53(20), 7749-7759. https://doi.org/10.1021/jf051513y

Vallverdu-Queralt, A., Regueird, J., Alvarenga, R.F.J., Huelamd, M.M., Leal, L.N. and Raventds, R.M.L. (2015). Characterization of the phenolic and antioxidant profiles of selected culinary herbs and spices: caraway, turmeric, dill, marjoram and nutmeg. Food Science and Technology, Campinas, $35(1), \quad 189-195 . \quad$ https://doi.org/10.1590/1678457X.6580

Womeni, H.M, Tonfack, D.F., Tiencheu, B. and Linder, M. (2013). Antioxidant potential of methanolic extracts and powders of some Cameroonian spices during accelerated storage of soybean oil. Advances in Biological Chemistry, 3, 304-313. https:// doi.org/10.4236/abc.2013.33034 